\title{
Aspetti epidemiologici e patogenetici dell'infezione da H.pylori
}

\section{Ida Luzzi}

Laboratorio di Batteriologia e Micologia Medica Istituto Superiore di Sanità

Viale Regina Elena, 299 - 0016 I Roma

\section{RELAZIONE}

Dal momento in cui Helicobacter pylori é stato isolato per la prima volta nel 1982 dalla mucosa gastrica di soggetti con gastrite (1), indagini clinico-epidemiologiche, istologiche e batteriologiche sono state condotte nel tentativo di determinare l'effettivo ruolo di questo microrganismo come agente causale di alcune patologie gastroduodenali. H.pylori è un microrganismo universalmente ubiquitario, la prevalenza dell'infezione varia da Paese a Paese raggiungendo anche il $90 \%$ nelle aree piu sottosviluppate del globo. E' ormai stabilito che il microrganismo è coinvolto nella patogenesi dell'ulcera peptica, duodenale e gastrica, del carcinoma e del linfoma gastrico $(2,3)$

A fronte dell'elevata prevalenza dell'infezione nell'uomo, solo una piccola proporzione di persone infette sviluppa ulcera peptica. Non si può quindi escludere l'esistenza di differenti proprietà di virulenza nell'ambito di diversi ceppi di H.pylori e tantomeno che fattori dell'ospite e fattori ambientali possano contribuire al diverso esito dell'infezione gastrica da parte di questo microrganismo (4-9)

Malgrado i numerosi studi effettuati, molti sono ancora gli aspetti da definire circa l'epidemiologia dell'infezione da H.pylori ed i meccanismi mediante i quali questo microrganismo é in grado di causare malattia

H.pylori è un batterio gram negativo microaerofilo che necessita di un lungo periodo di incubazione per svilupparsi in vitro. Specializzato a vivere in un unico ambiente, lo stomaco umano, H.pylo$r i$ va rapidamente incontro a trasformazioni morfo-fisiologiche quando viene a trovarsi in ambienti diversi. Tale trasformazione è rappresentata dalle cosiddette forme coccoidi, forme vitali ma non coltivabili. Da qui l'estrema difficoltà, se non l'impossibilità, di isolare H.pylori da campioni clinici diversi dalle biopsie gastriche, da campioni ambientali o da campioni di alimenti.

Anche se non del tutto chiarita, la trasmissione del microrganismo sembra avvenire da persona a persona attraverso la via oro-orale e oro-fecale, ma molto recentemente l'attenzione si è anche concentrata sulla possibilità che acqua ed alimenti, possano rappresentare importanti veicoli di trasmissione. Infatti grazie all'uso dell'amplificazio- ne genica e allo sviluppo di anticorpi monoclonali sono comparsi in letteratura lavori che hanno dimostrato la presenza di H.pylori in campioni di acqua superficiali e in alimenti di origine animale (10-12).

I determinanti di patogenicità presenti in tutti $\mathrm{i}$ ceppi di H.pylori sono essenzialmente quei fattori che consentono al microrganismo di colonizzare e di aderire all'epitelio gastrico. Tra questi i flagelli che conferiscono al batterio una vivace motilità, la produzione di ureasi che tampona l'acidità gastrica e le strutture di adesione del microrganismo giocano indubbiamente un ruolo di primaria importanza. Tra i fattori in grado di agire direttamente sui tessuti circostanti vanno citate una batterioferritina (NAP) in grado di attivare i neutrofili (13) e una citotossinatossina (VacA) che induce degenerazione vacuolare nelle cellule bersaglio attraverso l'inibizione della formazione delle membrane intracellulare (14-16)

A livello genetico esistono diverse forme alleliche del gene vacA (variazioni a livello della sequenza segnale e della regione mediana del gene) diversamente associate alla patologia

E' quindi possibile, mediante analisi per amplificazione genica, stabilire a quale genotipo vacA appartengono i ceppi di H.pylori isolati da pazienti con patologia gastroduodenale diversa, ed è stato possibile definire un'associazione significativa tra infezione da ceppi appartenenti al genotipo vacA s1 e ulcera peptica (tabella 1)(17-19).

Tra i determinanti di virulenza espressi solo da alcuni ceppi di H.pylori particolare importanza assume la cosiddetta isola di patogenicità cag (cag PAI) (21-23)

Si tratta di una sequenza di DNA contenente 31 geni il cui contenuto in $\mathrm{G}+\mathrm{C}$ risulta significativamente differente dal contenuto in $\mathrm{G}+\mathrm{C}$ del resto del genoma, a sostegno dell'ipotesi che la PAI sia stata acquisita da H.pylori durante il suo cammino evolutivo per trasferimento orizzontale da un'altra specie batterica ancora ignota. Alcuni dei geni contenuti nella PAI mostrano omologia con geni presenti negli operoni di altri batteri che codificano per un sistema di esporto di tipo IV specializzato nel trasferimento di complessi 
macromolecolari attraverso la membrana cellulare. Un marker della presenza della PAI è senza dubbio una proteina immunodominante a funzione sconosciuta, CagA. Gli isolati di H.pylori possono essere pertanto caratterizzati per la presenza della PAI attraverso 1'analisi di amplificazione genica specifica per il gene $\operatorname{cag} A$ o attraverso la rivelazione della proteina CagA mediante analisi in Western blot (tabella 2).

E' noto che durante il lungo periodo di infezione che H.pylori può causare esiste un'alternanza di fasi acute e fasi di remissione della patologia ulcerosa peptica. Ciò potrebbe essere spiegato dall'esistenza di un equilibrio dinamico tra popolazioni batteriche di H.pylori con e senza isola di patogenicità coesistenti a livello gastrico nello stesso individuo. La fase acuta corrisponderebbe ad una espansione della popolazione che possiede la PAI; questa, per effetto della pressione selettiva esercitata dall'ospite verso i ceppi che esprimono cagA, che come detto è un antigene immunodominante, potrebbe essere contratta a vantaggio dell'espansione della popolazione di H. pylori che ha perduto la PAI, con corrispondente remissione della patologia ulcerosa. Una nuova espansione della popolazione di H.pylori CagA positivi corrisponderebbe ad una fase di riacutizzazione della patologia (24).

Accanto alle proprietà patogenetiche del microrganismo, caratteristiche immunogenetiche dell'ospite possono incrementare il rischio d'infezione da $H p$ o di sviluppo delle patologie gastriche più gravi.

I polimorfismi del gene del complesso maggiore di istocompatibilità $H L A-D Q A 1$ e di alcune citochine proinfiammatorie quale 1'IL-1 (IL-1B e IL$1 R N$ ) sono coinvolti nel danno cellulare indotto da $H p$.

In conclusione una migliore comprensione della patogenicità dell'H.pylori e delle caratteristiche immunogenetiche dell'ospite nonché dei fattori ambientali che possono concorrere ad aumentare il rischio di infezione permetterà lo sviluppo di strategie volte alla riduzione della morbilità e della mortalità dovuta a due delle principali malattie gastroduodenali nel mondo ovvero 1'ulcera peptica ed il cancro gastrico. Infatti sebbene la scoperta dell'H.pylori abbia radicalmente modificato l'approccio medico verso tali patologie, l'eradicazione del microrganismo attraverso la terapia antibiotica è impraticabile per vasti gruppi di popolazione infetta. Questo, insieme alla comparsa di ceppi di H.pylori resistenti agli antibiotici, rende indispensabile lo sviluppo di strategie vaccinali sia per la terapia che per la profilassi dell'infezione da H.pylori.
Tabella I.

Associazione tra genotipo di vacA e patologia gastroduodenale

\begin{tabular}{lll}
\multicolumn{2}{c}{ Genotipo vacA } \\
\hline Pazienti & s I & s2 \\
\hline PUD & $80 \%$ & $20 \%$ \\
\hline NUD & $54 \%$ & $46 \%$ \\
\hline
\end{tabular}

PUD = ulcera peptica

NUD = dispepsia non ulcerosa

Tabella 2.

Associazione tra ceppi cagA positivi e patologia gastroduodenale

\begin{tabular}{lll}
\multicolumn{2}{c}{ cagA gene } \\
\hline Pazienti & presente & assente \\
\hline PUD $(n=101)$ & $79 \%$ & $21 \%$ \\
\hline NUD $(n=192)$ & $54 \%$ & $46 \%$ \\
\hline
\end{tabular}

$\mathrm{PUD}=$ ulcera peptica

$\mathrm{NUD}=$ dispepsia non ulcerosa

\section{BIBLIOGRAFIA}

1. Marshall BJ, Warren JR. Unidentified curved bacilli in the stomach of patients with gastritis and peptic ulceration. Lancet. 1984;1:1311-5.

2. Taylor DN, Blaser MJ. The epidemiology of Helicobacter pylori infection. Epidemiol Rev 1991; 13:42-59

3. Parsonnet J, Friedman GD, Vandersteen DP, Chang Y, Vogelman JH, Orentreich $\mathrm{N}$, et al. Helicobacter pylori infection and the risk of gastric carcinoma [see comments]. N Engl J Med 1991;325:1127-31 .

4. Mobley HL. Defining Helicobacter pylori as a pathogen: strain heterogeneity and virulence. Am. J. Med. 1997; 100:2S-11S

5. Atherton JC. The clinical relevance of strain types of Helicobacter pylori. Gut 1997;40:701-3

6. Axon, A.T. Are all helicobacters equal? Mechanisms of gastroduodenal pathology and their clinical implications Gut 199945 Suppl 1: 1-4

7. McGee, D.J.Mobley, H.L. Mechanisms of Helicobacter pylori infection: bacterial factors Current Topics In Microbiology And Immunology 1999 241: $155-80$

8. Covacci A, Telford JL, Del Giudice G, J. P, Rappuoli R. Helicobacter pylori virulence and genetic geography. Science 1999;284:1328-1333

9. Tomb JF, White O, Kerlavage AR, Clayton RA, Sutton GG, Fleischmann RD, et al. The complete genome sequence of the gastric pathogen Helicobacter pylori . Nature 1997;388:539-47

10. JP Hegarty, MT Dowd, KH Baker. Occurrence of H.pylori in surface water in the United States. J.App Microbiol 1999; 87:697-701;

11. Sasaki K et al. H. Pylori in the natural environment Scand J. Infect Dis 1999 31: 275-279;

12. Dore MP, Sepulveda AR, Osato MS, Realdi G, Graham DY. Helicobacter pylori in sheep milk. Lancet. 1999; 354: 132.

13. Evans DJ Jr, Evans DG, Takemura T, Nakano H, Lampert HC, Graham DY, Granger DN, Kvietys PR. Characterization of a Helicobacter pylori neutrophilactivating protein. Infect Immun. 1995 22:13-20. 
14. Graham MF, Blaser MJ, et al. Helicobacter pylori cytotoxin induces vacuolation of primary human mucosal epithelial cells. Infect Immun 1996;64:486771

15. Lupetti P, Heuser JE, Manetti R, Massari P, Lanzavecchia S, Bellon PL, Dallai R, Rappuoli R, Telford JL. Oligomeric and subunit structure of the Helicobacter pylori vacuolating cytotoxin.J Cell Biol. 1996;133:801-7.

16. Reyrat JM, Pelicic V, Papini E, Montecucco C, Rappuoli R, Telford JL.

Towards deciphering the Helicobacter pylori cytotoxin.

Mol Microbiol. 1999; 34:197-204.

17. S.R. Han Schreiber, H.J.Bhakdi, S.Loos, M.Maeurer, M.J. vacA genotypes and genetic diversity in clinical isolates of Helicobacter pylori Clinical And Diagnostic Laboratory Immunology1998; 85 139-45.

18. Cover TL, Tummuru MK, Cao P, Thompson SA, Blaser MJ. Divergence of genetic sequences for the vacuolating cytotoxin among Helicobacter pylori strains. J Biol Chem 1994;269:10566-73

19. Atherton JC, Cao P, Peek RM, Jr., Tummuru MK, Blaser MJ, Cover TL. Mosaicism in vacuolating cytotoxin alleles of Helicobacter pylori. Association of specific vacA types with cytotoxin production and peptic ulceration. J Biol Chem 1995;270:17771-7

20. Rappuoli, R.Lange, C.Censini, S.Covacci, A. Pathogenicity island mediates Helicobacter pylori interaction with the host Folia Microbiologica 1998; 43:275-8

21. Covacci A, Falkow S, Berg DE, Rappuoli R. Did the inheritance of a pathogenicity island modify the virulence of Helicobacter pylori? Trends Microbiol. 1997 ;5:205-8

22. Censini S, Lange C, Xiang Z, Crabtree JE, Ghiara P, Borodovsky M, et al. cag, a pathogenicity island of Helicobacter pylori, encodes type I- specific and disease-associated virulence factors. Proc Natl Acad Sci U S A 1996;93:14648-53

23. Akopyants NS, Clifton SW, Kersulyte JE, Crabtree $\mathrm{JE}$, Youree BE, Reece CA, et al. Analyses of the cag pathogenicity island of Helicobacter pylori. Mol. Microbiol. 1998;28:37-54

24. Covacci A, Rappuoli R. Helicobacter pylori: molecular evolution of a bacterial quasi-species.Curr Opin Microbiol. 1998 1:96-102.

25. Carattoli, C. Pezzella, A Pietroiusti, A. Galante, P. Pezzotti \& I. Luzzi Cytotoxin associated gene A and vacuolating cytotoxin A in human isolates of Helicobacter pylori and their association with the clinical status of ulcer disease.2000. Eur. J. Gastroenteroly 12: 1207-1213

26. Xiang Z, Bugnoli M, Ponzetto A, Morgando A, Figura N, Covacci A, et al. Detection in an enzyme immunoassay of an immune response to a recombinant fragment of the 128 kilodalton protein (CagA) of Helicobacter pylori. Eur J Clin Microbiol Infect Dis 1993;12:739-45

(Azuma T. et al. , 1994, Lancet 343: 542;

El-Omar EM et al, 2000, Nature 404:398). 